\title{
Planet masses and radii from physical principles
}

\author{
Guenther Wuchterl ${ }^{1}$ \\ 1 Thüringer Landessternwarte, D-07778 Tautenburg, Germany \\ email: gwuchterl@tls-tautenburg.de
}

\begin{abstract}
Masses and radii are the primary observables to characterize exoplanets today. A self-consistent theoretical approach is presented that allows to calculate mass- and radiusdistributions of exoplanet populations from basic physical principles and avoids the usual parametrisation of a multitude of processes.
\end{abstract}

Keywords. planetary systems: formation

\section{Planet formation theory for the discovery era}

An era of discovery in astronomy puts theory in a mixed situation: on the one hand new key objects are discovered that allow to adjust incompletely understood parts of the theories, on the other hand the nature of what is found may be severely determined by the selection bias that is ubiquitous at the frontier of astronomical endeavors. One approach in this situation is to try to work out as much as possible from the most basic principles, the ones that are the best tested. The results may be limited but they provide solid ground and help the discovery process itself. To prepare for the CoRoT mission Pečnik (2003); Pečnik (2005); Pečnik \& Wuchterl (2005); Broeg (2006); Broeg (2009); Schönke (2005); Schönke (2007); and Wuchterl et al. (2007) worked out a theory that is based on similar principles and tools as the theory of stellar structure and evolution. It provides statistical information on the masses and radii of exoplanets.

\section{Heuristics - in search of principles}

The motivation and idea is twofold: (1) the stellar main-sequence provides understanding of the properties of most stars for most of the time without knowing the details of their formation process. The statistics of stars in the Hertzsprung-Russel diagram is dominated by evolutionary time-scales derived from stellar evolution physics, (2) detailed, fluiddynamical calculations of planet formation showed convergence towards certain classes of planets (Wuchterl 1995; Wuchterl et al. 2000). Of course strong assumptions are necessary to work out a prediction from basic principles. For the stellar population and its main sequence these are the well known equilibria of stellar structure: hydrostatic and thermal equilibrium. While they are motivated by time-scale arguments their use is more a pragmatic assumption than a strict mathematical derivation. In all cases there is an implicit underlying assumption: the material manages to assemble into a star and the composition is the one that is observed. To construct an analogon, a planetary main sequence we first note that planets as stars are hydrostatic most of their time, a fact that is even part of the 2006 IAU definition. Secondly, while stars are in thermal equilibrium on the main sequence, planets do not have sufficient energy sources to sustain thermal equilibrium during most of their live. During formation, however they have significant energy 
resources, from contraction and planetesimal accretion. That leads to near equilibrium conditions in the protoplanets during most of their growth time (e.g. Wuchterl 1993 for a quantification of 'near' by radiation fluid-dynamics). Thus if we study the properties of the planetary equilibrium conditions during the formation era, we can estimate the typical properties of planets. After all, the planets have to grow through some of these equilibria and certainly end up near one of them, after dynamical events have settled down. It is interesting to note that planets, unlike stars are in or near thermal and hydrostatic equilibrium during a time when they are still embedded in the mass-reservoirs from which they are accumulated and are then able to exchange mass with the environment, i.e. the protoplanetary nebula. Thus there is some prospect to derive information on the typical mass of a planet from a study of planetary equilibria.

\section{General theory of planet formation - assumptions and strategy}

The goal is to build a theory for all detectable planets. Thus it should be, (a) general - for all stars and all orbital periods, (b) simple - with a direct relation to physics, (c) robust - with no a-priori selection of mechanisms, (d) predictive - contain no free parameters, (e) fast - make evaluation feasible to better than observed accuracy in the entire discovery space.

To calculate specific results in such a general approach we have to make strong assumptions to facilitate the task. We assume: (1) the diversity of nebulae: study planet formation for all gravitationally stable protoplanetary nebulae around stars of all masses; (2) the strong planetesimal hypothesis: there are always enough planetesimals; (3) the impossibility to chose a particular 'scenario' now and study all planets with cores of all masses including zero - we do not separate planets formed by nucleated instability from others, perhaps formed by a disk instability.

\section{Isothermal planets and planetary mass}

To understand the key physical properties of the planetary equilibria Pečnik (2003) first studied a simple model in detail - isothermal planets. He constructed the hydrostatic equilibria of an isothermal ideal gas around a core. The planetary envelope was embedded in the nebula inside the Hill-sphere at given distance from a star. Pečnik introduced a planetary 'phase diagram' and found that all planets fall in one of four classes in the core-mass, core-surface-density diagram: (I) mature terrestrial planets, (II) mature giant planets, (III) 'rock in the fog', (IV) protoplanets (Pečnik \& Wuchterl 2005). The structure of the diagram being centred around a generalised critical core mass for planets. Schönke (2005) showed which of the isothermal planetary equilibria are stable and could find an analytical expression for the characteristic mass - close to but not identical with the critical one - and density, $M_{\Omega}, \varrho_{\Omega}$, resp., shaping the entire diagram, and thus defining a scale for planetary mass (Schönke 2007):

$$
M_{\Omega}=\left(\frac{\frac{k_{\mathrm{B}} T}{G m_{\mathrm{molecular}}} \ln \left(\frac{4 \pi a^{3} \varrho_{\text {core }}}{27 M_{\text {star }}}\right)}{\left(\frac{4 \pi \varrho_{\text {core }}}{3}\right)^{\frac{1}{3}}-\frac{\left(3 M_{\text {star }}\right)^{\frac{1}{3}}}{a}}\right)^{\frac{3}{2}} ; \varrho_{\Omega}=\frac{\varrho_{\text {core }}}{3} .
$$

This expression gives a natural planetary mass derived from micro-physics - molecular mass, $m_{\text {molecular }}$, primary mass, $M_{\text {star }}$, planetary core density, $\varrho_{\text {core }}$ and orbital radius, $a\left(G\right.$ and $k_{\mathrm{B}}$ are the gravity and Boltzmann-constants, $T$, the temperature of planet and nebula gas). The planetary equilibria turned out to be governed by the self-gravity of the 
planetary envelopes in an interplay with the stellar tides forming the Hill-sphere (app. the Roche-Lobe in this regime). Planet formation can accordingly be understood as a compactification of a tenuous protoplanet to a dense planet driven by self-gravity.

\section{Mass spectra for planets with energy transfer}

The critical masses for isothermal planets are hundred times smaller than those of realistic planets because the inner parts of the envelopes become usually opaque and are heated significantly before the critical mass is reached. The temperature gradients due to planetesimal accretion require a treatment of energy transfer by radiation and convection as well as the use of realistic equations of state to account for the incompressible, liquidlike behaviour in the deep planetary interior. Broeg (2006), Broeg (2007b), Broeg (2009) constructed such planets for host stars of 0.4 to 2 solar masses and orbital periods of 1 to 64 days to estimate the planetary mass function in the discovery window of CoRoT. Results are available online for $\mathrm{M}$ to A stars and orbital periods up to 128 days: CoRoT - Mark 1: Dec. 2005; Mark 2: Sept. 2007; Mark 3: June 2008 (Broeg 2007a). These planetary mass spectra are typically bimodal with peaks near Jupiter's and Neptune's mass and they show a rich dependence on host star mass and orbital period, cf. Broeg (2007b), Broeg (2009).

\section{The CoRoT pre-launch planetary mass function prediction}

Two extra steps are necessary to derive epoch of observation mass functions that a planet search would find on the sky: (1) the stability of the planetary equilibria has to be investigated and (2) possible effects of mass loss during the planetary evolution into the present have to be estimated. On Dec. 26th, just before the launch of the CoRoT-satellite on Dec. 27th, 2006, Wuchterl et al. (2007), Lammer et al. (2007) made these estimates based on the above mass spectra, an isothermal stability analysis (Schönke 2005), and, for test-cases, detailed quasi-hydrostatic and non-linear, radiation fluid dynamical calculations. The main features of the resulting theoretical planetary mass functions for a stellar field, are a dominating population of Hot Neptunes and a double peaked mass function, with peaks near Jupiter's and Neptune's mass, cf. Fig. 1. The mass-distribution is probably robust against mass loss for periods starting at $4 \mathrm{~d}$, cf. Lammer, this volume. For high resolution, extended period range and analysis of the underlying physics that sometimes leads to a triple-peak structure, see Broeg (2009).

\section{Theoretical planet radii and transit signals}

The above mass distributions do not contain any history, the are derived from the statistics of self-gravitating gas spheres that are embedded into protoplanetary nebulae with a finite but sometimes very small pressure. They are best viewed as initial planetary mass functions before and until the planets detach from the nebula, which acts as a massand thermal reservoir.

The evolution into the present, towards the epoch of observation, needs to be calculated to determine the corresponding planetary radii. That involves an assumption about how the nebula that corresponds to a given planet disappears and how the energy supply of the planet by planetesimal accretion decays. For simplicity and comparability with conventional planetary evolution calculations we assume that the nebula is dissipated, and the planetesimal accretion decays with a Ma time-scale and that the mass of a planet remains constant. Accordingly for every planet the nebula pressure at its 

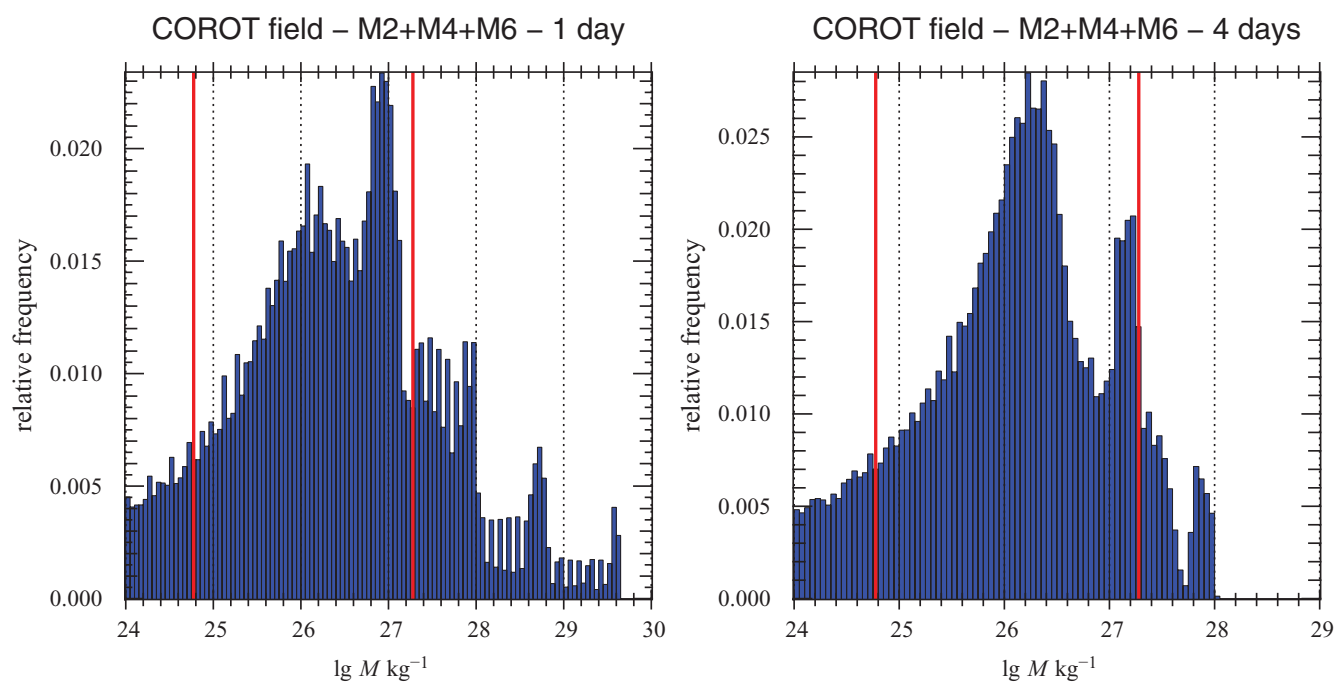

Figure 1. Pre CoRoT launch prediction (Dec. 2006) of the planetary mass function of a stellar population mix typical for a CoRoT field and planetary orbits of 1 (left) and 4 days. The relative frequency of planets is plotted vs. the decadic logarithm of planetary mass, thick (red) vertical lines mark Earth and Jupiter mass, resp., see Wuchterl et al. (2007).

Hill-sphere is exponentially lowered and the energy source due to planetesimal accretion is shut off. Thus every planet undergoes a decompression that is fast but on a thermal time-scale. Initially that is a test for secular (thermal) stability as the planet responds to being exposed to vacuum. As the contraction and cooling proceeds, the computation approaches a classical planetary evolution calculation with a plausible initial condition. Guaranteeing this convergence is the main motivation for keeping the mass constant. The key difference to conventional evolutionary calculations is that the initial conditions are diverse, with many protoplanets exhibiting a relatively high density trough-out their Hill-sphere. The broad spectrum of planets with its variety in core mass, nebula pressure and planetesimal accretion-rates assures that every physically possible evolution is calculated. Technically this is a pre-main sequence-like, quasi-hydrostatic calculation of
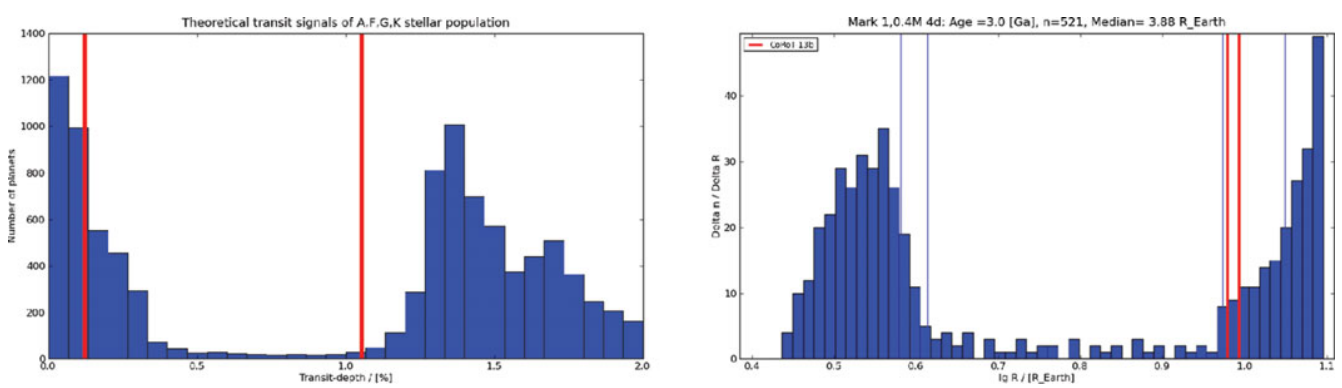

Figure 2. Left: Typical distribution of transit signals obtained from planetary evolutionary calculations for a sample planets orbiting A, F, G and K host stars with periods < $50 \mathrm{~d}$. Right: Distribution of the planetary radii at an age of $3 \mathrm{Ga}$, calculated for the the CoRoT-13b orbital position. Dark (red) lines mark the radii consistent with the CoRoT-transit-light curve. Light (blue) lines mark the radii of solar system giant planets.

a radiative/convective gas shell (the planetary envelope) around a rigid core. The planet radiates into the radiation field of the star at its orbital radius. Thus irradiation by 
the star is automatically accounted for. To obtain the radius of the planet the optical depth is integrated inward until $2 / 3$ is reached - the planetary photosphere. Thus the photospheric, more specifically the optical depth radius is used as the planetary radius here.

Once the calculations have reached a given age we do statistics of the radii and obtain the planetary radius function for that age. Taking the squared ratio of the planet radius to its host star radius we obtain the transit signal-strength. We built a sample of all these ratios for all the planetary models at a given age for any given initial mass spectrum, i.e. host star mass, orbital period and planetesimal accretion. Then, summing over all the results across the stellar masses and the $1-64 \mathrm{~d}$ period range we obtain the distribution of transit signals expected at the given age. We see a result for stars of spectral types $\mathrm{K}$ to A relevant for a CoRoT long run in Fig. 2, left. The bi-modality of the planetary mass functions is enhanced by the fact that smaller planets tend to have a larger core and thus higher density. This produces a distribution of transit signals with two peaks and a large gap in between. The theoretical statistics of planets thus shows that one is likely to first detect large 'Jupiters' with a signal strength in the \%-range and has to reach a precision of $\sim 0.1 \%$ (half maximum) and to enter the Neptune-regime to detect smaller planets with some probability. As a test of theory we confront it to CoRoT-13b (Cabrera et al. 2010), with its $\sim 200$ earth-mass heavy element core (Fig. 2, right). The extreme planet is located somewhat towards the lower end of the Jupiter-peak in the radius distribution but still predicted to occur relatively frequent.

\section{Outlook - the statistical planetary mass-radius diagramm}

The high resolution mass- and radius distributions allow to study the theoretical frequency of planets in the mass-radius diagram for a given age. A sample calculation for the planets of a solar mass star at $1 \mathrm{Ga}$ with orbital periods of 1 and 64 days is shown in Fig. 3 together with known exoplanets (Oct. 2010).

\section{Conclusion - results from the general theory of planet formation}

By assuming a diversity of stable protoplanetary nebulae and that there are always enough planetesimals to build condensible element cores when required, a statistical approach to planets can explain the values of planetary masses for a given stellar mass and orbital radius. A-priori there are no assumptions made about the necessity of a core for a planet and where the planet was located in its past. The theory is readily applicable to all stellar masses and orbital radii, for planets with and without a core. In that sense it is general. It predicts bimodal mass and radius distributions with peaks near Jupiter's and Neptune's properties. The 'Neptunes' are always dominating by number. The statistics of transit signals shows a large gap between Jupiters and Neptunes. Mass and radius of extreme planets as CoRoT-13b with its $\sim 200$ earth-mass core can be explained as physically possible with a nebula origin and likely for the age of its host star and conditions at its orbit. The possibility to evaluate the mass- and radius distributions relatively fast allows a population-density approach to the planetary mass-radius diagram. Locations in the diagram that contain large numbers of physically possible objects with a nebula origin, and thus according to this theory likely planets of a given age, can be used much like isochrones in the conventional HRD or similar diagrams. The isochrones of stellar structure become two-dimensional distributions of planetary properties for given age. 
Mark 1 R, 1-64d; Age $=1.000[\mathrm{Ga}]$

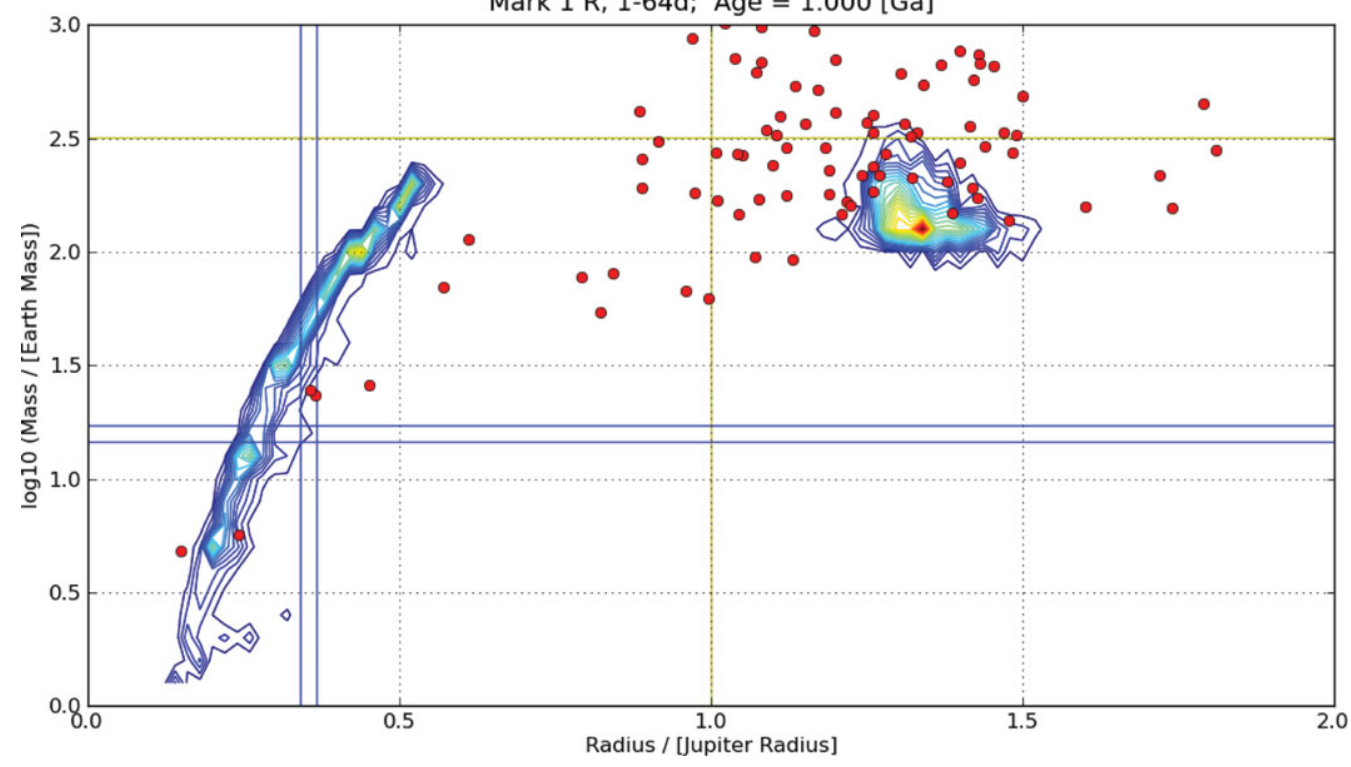

Figure 3. Theoretical population density (shaded contours) in the mass-radius diagram: initial mass functions from the statistics of core-envelope equilibria embedded in diverse protoplanetary nebula and subsequent planet-evolution calculations to determine the radii as a function of time. Shaded-contours point to many planets. Note the branch of core-dominated 'Neptunes' towards the left and the clump of 'Jupiters' in the upper right. Superimposed are exoplanets (dots) and Jupiter, Uranus and Neptune (lines).

\section{References}

Broeg, C. 2006, Ph.D. thesis, Friedrich-Schiller-Universität Jena, Germany. http://www.dbthueringen.de/servlets/DocumentServlet?id=6619

- 2007a, Mass spectra corot mark 2a. Http://www.space.unibe.ch/ broeg, http://www. space.unibe.ch/ ${ }^{\sim}$ broeg

- 2007b, MNRAS, 377, L44

Broeg, C. H. 2009, Icarus, 204, 15

Cabrera, J., Bruntt, H., Ollivier, M., et al. 2010, A\&A, 522, A110+

Lammer, H., Penz, T., Wuchterl, G., et al. 2007, ArXiv Astrophysics e-prints. arXiv:astro$\mathrm{ph} / 0701565$

Pečnik, B. 2005, Ph.D. thesis, Ludwig-Maximillians-Universität München, Germany. http://edoc.ub.uni-muenchen.de/archive/00005023/01/Pecnik_Bojan.pdf, http://www.znanost.org/ bonnie/work/Thesis.pdf

Pečnik, B. \& Wuchterl, G. 2005, A\&A, 440, 1183

Pečnik, B. 2003, Master's thesis, University of Zagreb, Croatia

Schönke, J. 2005, Diploma Thesis, AIU-Jena

Schönke, J. 2007, Plan. \& Sp. Sci., 55, 1299

Wuchterl, G. 1993, Icarus, 106, 323

- 1995, Earth, Moon and Planets, 67, 51

Wuchterl, G., Broeg, C., Krause, S., et al. 2007, arXiv:astro-ph/0701003

Wuchterl, G., Guillot, T., \& Lissauer, J. J. 2000, Protostars and Planets IV, 1081 